\title{
Enhancing proficiency in performing endoscopic submucosal dissection (ESD) by using a prototype robotic endoscope
}

Authors

Institutions
Philip WaiYan Chiu', Soo Jay Phee ${ }^{2}$, Pradeep Bhandari ${ }^{3}$, Kazuki Sumiyama ${ }^{4}$, Tomohiko Ohya ${ }^{4}$, Jennie Wong ${ }^{6}$, Carmen CY Poon ${ }^{1}$, Hisao Tajiri ${ }^{4}$, Kiyokazu Nakajima ${ }^{5}$, Khek Yu Ho ${ }^{6,7}$

Institutions are listed at the end of article. submitted 9. March 2015 accepted after revision 26. June 2015

\section{Bibliography}

Dol http://dx.doi.org/ 10.1055/s-0034-1393178

Published online: 14.10 .2015

Endoscopy International Open 2015; 03: E439-E442

(c) Georg Thieme Verlag KG Stuttgart . New York E-ISSN 2196-9736

\section{Corresponding author}

\section{Khek Yu Ho, MD}

Department of Medicine Yong Loo Lin School of Medicine National University of Singapore Singapore

Fax: +65-67794112

khek_yu_ho@nuhs.edu.sg
Background and study aims: One of the challenges in performing endoscopic submucosal dissection (ESD) is the lack of counter traction during submucosal dissection. MASTER (Master and Slave Transluminal Endoscopic Robot) was designed to allow performance of complex endoluminal procedures using two arms with excellent control. This study aimed to compare the performance of ESD between endoscopists and novices using MASTER.

Methods: This is a prospective study comparing the differences in performing ESD using MASTER in an ex vivo porcine stomach model among individuals with or without experience in surgery and endoscopy. Multiple standardized lesions of $20 \mathrm{~mm}^{2}$ were pre-marked on an ex vivo porcine stomach. Each participant received basic training in controlling MASTER before the ESD procedure. The operative time and size of specimen obtained by each participant were noted.

Results: Nine individuals (three ESD expert endoscopists, three ESD non-expert endoscopists,

\section{Introduction}

$\nabla$

Gastrointestinal cancers remain the most common and important cancer worldwide [1]. Early diagnosis of gastrointestinal cancers will lead to improvement in clinical outcomes and survival. For early gastrointestinal cancers with minimal risk of lymph node metastasis, endoscopic resection is the current standard of treatment [2]. Endoscopic resection with the technique of endoscopic submucosal dissection (ESD) achieved a high rate of en bloc resection. Retrospective cohort studies showed that ESD reduced the rate of local recurrence when compared to endoscopic mucosal resection (EMR) $[3,4]$. Despite the advantages in oncological clearance, ESD is technically challenging. A study on the learning curve for ESD showed that the en bloc resection rate increased from $45 \%$ to $85 \%$ after the first 40 cases, and three non-clinician novices) participated in the experiment. All participants completed the ESD procedure for en bloc resection of standardized lesions using EndoMASTER without perforation. The mean times (mean \pm SD) taken by ESD expert endoscopists, ESD non-expert endoscopists, and novices to complete Robotic ESD were $122 \pm 58 \mathrm{~s}, \quad 203 \pm 150 \mathrm{~s}$, and $561 \pm 496 \mathrm{~s}$, respectively. There was no statistically significant difference in the mean operative time to complete the ESD between the three groups $(P=0.242)$. When the performance of the six endoscopists was compared to that of the three novices, the endoscopists took an average of $162 \pm 111 \mathrm{~s}$ to complete the ESD, while the non-clinicians required an average of $561 \pm 496 \mathrm{~s}(P=0.085)$.

Conclusions: There was a trend to shorter operative time when comparing endoscopists to nonendoscopists in performing ESD using MASTER. The use of MASTER enabled the novice without endoscopy experience to complete the ESD procedure.

while the perforation rate decreased after the initial 20 cases [5].

We conducted a study of the preclinical training for experienced endoscopists to perform ESD in a porcine model [6]. Despite their vast experience in esophagogastroduodenoscopy (EGD), colonoscopy, and endoscopic retrograde cholangiopancreatoscopy (ERCP), 62\% of the participants achieved a perforation upon first performance of ESD. The development of Master and Slave Transluminal Endoscopic Robot (MASTER) aimed to improve the performance of complex endoluminal procedures by mounting two robotic arms onto an ordinary double channel endoscope [7]. A multicenter prospective cohort study had confirmed the feasibility and safety in performing ESD using MASTER to treat early gastric cancer in five patients [8]. The current study aimed to investigate the enhancement of proficiency in performing 


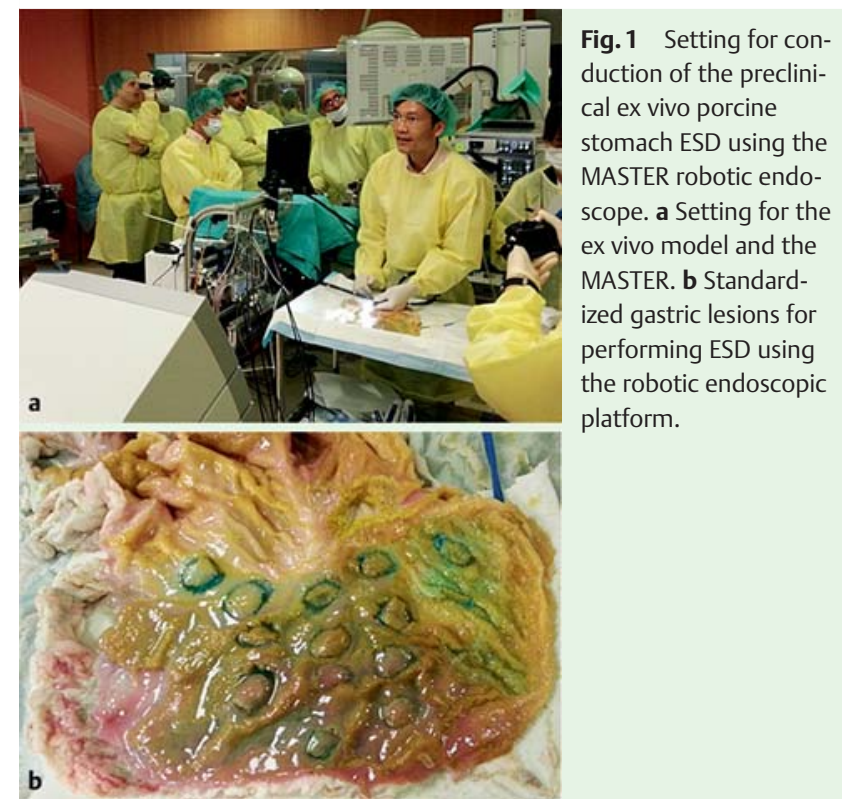

ESD using MASTER through a comparison of ESD experts, ESD non-experts, and novices in an ex vivo porcine stomach model.

\section{Method \\ $\nabla$}

This is a prospective preclinical study investigating the effects of use of a prototype robotic endoscope on the proficiency of performing ESD in an ex vivo porcine stomach model. Three groups of participants were invited to perform ESD using the Master and Slave Transluminal Endoscopic Robot (MASTER). The first group consisted of ESD expert endoscopists who had performed more than 100 cases of ESD. The second group consisted of ESD nonexpert endoscopists with vast experience in performing esophagogastroduodenoscopy (EGD), colonoscopy as well as endoscopic retrograde cholangiopancreatoscopy (ERCP). They either had experience with less than 10 cases of ESD or had never performed the procedure. The third group consisted of novices who had never performed any endoscopic procedure. They were engineers involved in material design not related to endoscopy.

All of the procedures were performed using the MASTER endoscopic robot which was held by a single endoscopist (KYH) experienced in handling the MASTER robot ( $\bullet$ Fig. 1$)$. In order to standardize the size of the lesion for the trial, we created a standard $20 \mathrm{~mm}$ circular cardboard disc which was then used to create standard $20 \mathrm{~mm}$ circular lesions with diathermy markings (ERBE 300 D, ERBE, Germany) in an ex vivo porcine stomach. After submucosal injection of normal saline mixed with indigo carmine, a circumferential mucosal incision was made using an IT2 knife (Olympus Co Ltd, Japan) ( $\bullet$ Fig. 1). The study started after completion of the circumferential mucosal incision. All participants completed the ESD procedure using the MASTER robot ( $\bullet$ Fig. 2). The steps for performance of ESD were standardized as: 1. Grasping of the mucosa using the right arm of the MASTER; 2. Elevation of the mucosa after grasping to expose the submucosal plane; 3.Submucosal dissection using the left arm with the diathermy hook; and 4. Repeating the procedure until complete dissection of the gastric lesion had been achieved.

Each of the participants performed three ESD procedures, and all procedures were video-recorded and timed. The outcomes in-



Fig. 2 a External view demonstrating submucosal dissection using the MASTER robotic endoscope. $\mathbf{b}$ Endoscopic view showing lifting of mucosa and submucosal dissection using the MASTER robotic endoscope.



(2)

cluded the time for first successful grasp of the mucosa, time to achieve adequate elevation and exposure of the submucosa, time for performance of submucosal dissection, and total time for the procedure. We recorded all perforations during the procedure. This study was approved by National University of Singapore Institutional Animal Care and Use Committee (IACUC protocol no: 024/12).

All of the data were analyzed using SPSS version 11.0. The analysis of continuous data was performed using the Student's $t$ test, while the categorical data were analyzed with the Chi-squared test. Intergroup comparison was conducted using one-way ANOVA. We defined statistically significant results as having a $P$ value less than 0.05 .

\section{Results}

$\nabla$

A total of nine participants completed the ESD procedures for resection of the standardized $20 \mathrm{~mm}$ lesion using the MASTER endoscopic robot. Table 1 shows the baseline demographics for the different groups of participants. The ESD expert group consisted of one surgeon and two gastroenterologists. The ESD non-expert endoscopists group consisted of two surgeons and one gastroenterologist. The non-clinician novice group consisted of three engineers focusing on material design and development without experience in performing endoscopy. Table 1 illustrates that the endoscopists in the ESD expert group were more experienced and had performed many more endoscopic procedures than the ESD non-expert group of endoscopists.

All participants were able to complete the ESD procedures and there were no perforations during the procedure. When the operative times were compared between the three groups, there was no difference in the time for first grasping of mucosa, time to expose the submucosa, time for submucosal dissection, and total time for the procedure ( $\bullet$ Fig. 3 ). There was a trend towards longer operative time for the non-clinician novice to complete the submucosal dissection though the difference was not statistically significant. The average operative time for MASTER ESD was $121.7 \pm 57.7$ s for the ESD expert group, $202.7 \pm 149.5$ s for the ESD 
Table 1 Baseline demographics for the participants.

\begin{tabular}{|c|c|c|c|c|}
\hline Participant groups & $\begin{array}{l}\text { Years as specialist endoscopist } \\
\text { (Gastroenterologist/Surgeon) }\end{array}$ & $\begin{array}{l}\text { No. of EGD } \\
\text { performed }\end{array}$ & $\begin{array}{l}\text { No. of colonoscopies } \\
\text { performed }\end{array}$ & $\begin{array}{l}\text { No. of ERCP } \\
\text { performed }\end{array}$ \\
\hline ESD expert endoscopists & 12.67 & 10666.7 & 5000.0 & 438.0 \\
\hline ESD non-expert endoscopists & 12.33 & 6766.7 & 2266.7 & 333.3 \\
\hline Novices & 0 & 0 & 0 & 0 \\
\hline
\end{tabular}

EGD, esophagogastroduodenoscopy; ERCP, endoscopic retrograde cholangiopancreatoscopy.

ESD expert group consisted of one surgeon and two gastroenterologists; ESD non-expert group consisted of two surgeons and one gastroenterologist; Novice group consisted of engineers without experience in performing endoscopy.

non-expert group, and $561.0 \pm 495.6$ s for the novice group $(P=$ 0.242 ) ( Fig.4). When all the clinicians were grouped to compare with the non-clinicians, we found that there was a trend towards a longer total dissection time $(P=0.085 ; 95 \% \mathrm{CI}=-70.8$ to $868.5)$ and submucosal dissection time $(P=0.078 ; 95 \% \mathrm{CI}=-48.5$ to 705.8 ) for the non-clinician group as compared to clinician group, however these differences were not significant.

\section{Discussion}

$\nabla$

Gastrointestinal cancers are important causes of cancer worldwide [1]. Colorectal, gastric, and esophageal cancers ranked the second, fourth, and fifth most common cancers in Asia [9]. The majority of gastrointestinal cancers are diagnosed at late stage with grave prognosis despite major surgery and oncological therapies. On the other hand, early stage gastrointestinal cancers carry excellent prognosis with 5-year survival of over $85 \%$ if treated appropriately [10]. Intramucosal early gastrointestinal cancers can be treated by endoscopic resection with adequate oncological clearance due to minimal risk of lymph node metastasis [11]. ESD has emerged as a safe and effective method of endoscopic resection with a high rate of en bloc resection. Recently, ESD is being increasingly practiced in countries outside Japan and Korea. A European series reported 82 cases of epithelial or submucosal lesions treated with ESD [12]. The en bloc resection rate was $77.1 \%$, while the local recurrence was $0 \%$ after R0 resection compared to $38.5 \%$ after piecemeal resection. Although the procedural time per specimen size was significantly shorter in the second half of the study, the authors commented that ESD was difficult, time consuming, and required a prolonged learning curve.

One of the reasons for the difficulties in ESD is the lack of appropriate endoscopic instruments and platform for performing the dissection. Unlike minimally invasive surgery where surgeons performed dissection using two instruments under the laparoscope in a triangulation manner, ESD is performed in a single handed manner with the instrument co-axially aligned with the endoscope. The advantages of the Da Vinci Robotic surgical system was clearly demonstrated in performing complex surgical tasks within a confined space during radical prostatectomy [13]. The development of the MASTER aimed to improved surgical dissection within the gastrointestinal lumen through a flexible endoscopic platform mounted with two robotic arms [7,14].

The current study employed the first generation of MASTER robotic endoscope which has two robotic arms, one with a dissection hook and the other with a forceps attached to an ordinary dual channel endoscope. Our results demonstrate that the techniques and outcomes of ESD can be standardized using the MASTER. Standardization of ESD technique using devices such as the MASTER raises hope for a large number of endoscopists world-

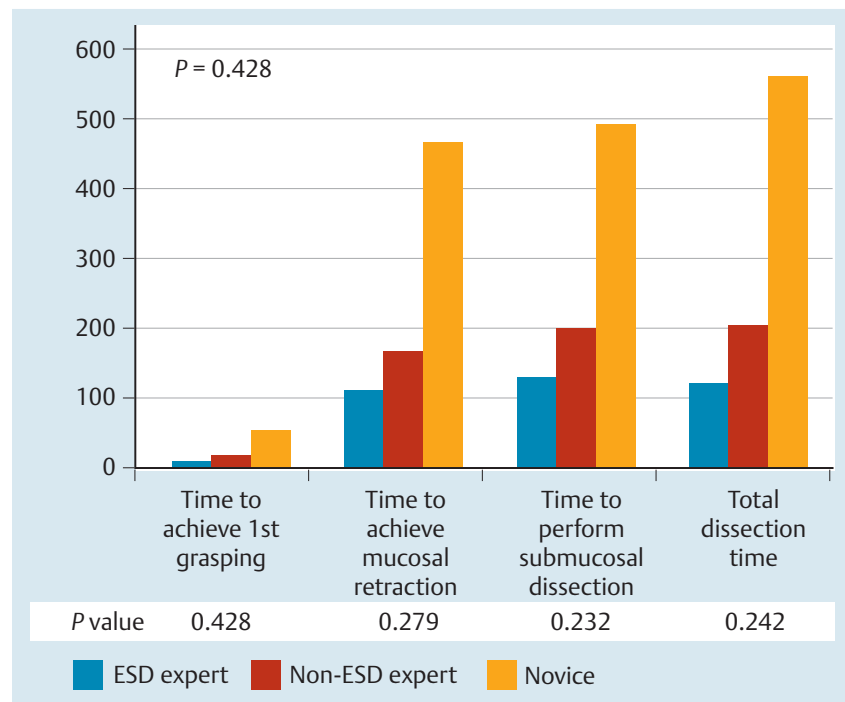

Fig. 3 Comparison of the operative time (s) for MASTER ESD among ESD experts, non-experts, and novice study groups.

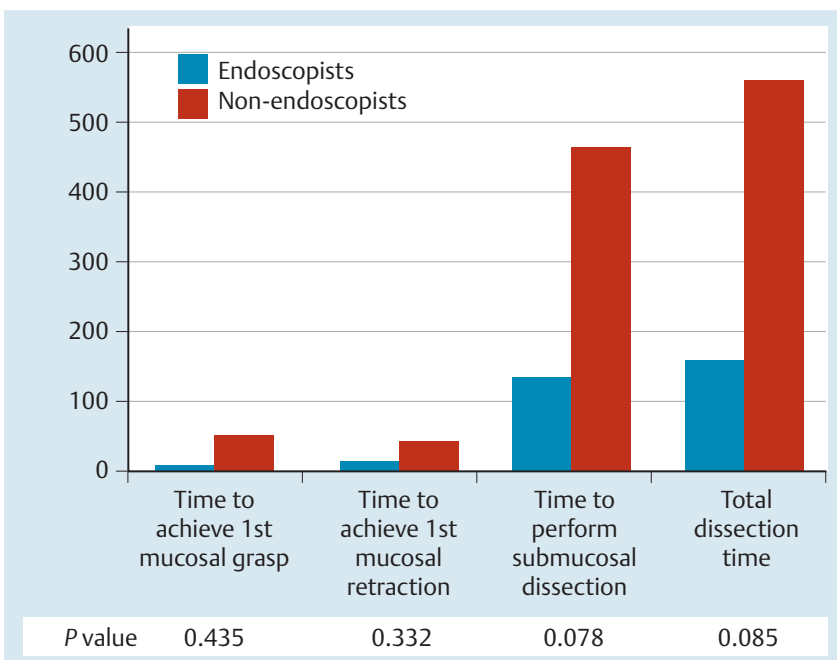

Fig. 4 Comparison of the operative time (s) for MASTER ESD between endoscopist and non-endoscopist study groups.

wide to practice ESD, who may find the procedure to be technically challenging, requiring prolonged procedural times, and associated with a high complication rate. The other challenge facing the widespread applicability of ESD is the learning curve. It is well known that this can be very long especially in centers with a small case load and few experts. In our study, the non-clinician group with no experience in endoscopy managed to grasp the concept of ESD and complete the procedure within $20 \mathrm{~min}$. This 
Video 1

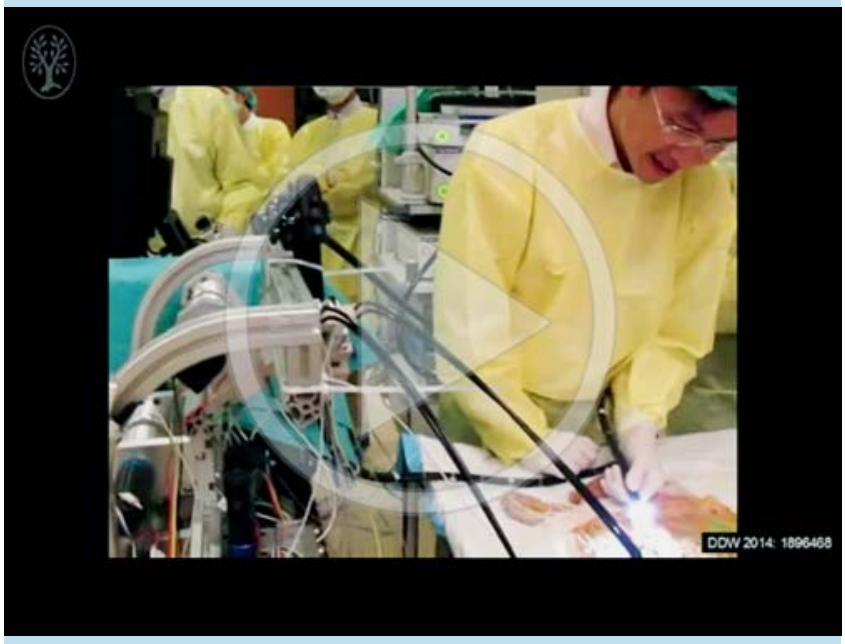

Using the prototype MASTER endoscopic robotic platform. Comparison between expert and novice endoscopists. Online content including video sequences viewable at: http://dx.doi.org/10.1055/s-0034-1393178

may suggest that devices such as the MASTER can significantly shorten the learning curve for ESD.

The procedure for ESD was greatly enhanced by using the two end effectors for dissection, one of the aims being to attain adequate exposure of the submucosal plane with the other aim being safe dissection. As participants in the novice group were engineers with no previous experience in performing endoscopy or ESD, robotic technology is one of the methods used to overcome inadequate experience and learning curve. This is important evidence to demonstrate that flexible robotic technologies will enhance the performance of complex dissection within the gastrointestinal lumen.

This study was limited by several factors. The model employed was an ex vivo porcine stomach, hence bleeding during ESD could not be simulated. Moreover, the size of the lesion was small, which might not be representative of a true gastric ESD, especially from the viewpoint of risk of perforation. The number of participants and the number of MASTER ESD procedures performed were limited. As it was an ex vivo model study, the results may not be directly applicable to human patients. The prototype MASTER robotic system also had limitations. In the clinical setting, one of the major limitations was the inability to interchange the two robotic arms. This led to the need for twisting and torque of the endoscope to facilitate dissection. We can expect future refinement of the MASTER robot before clinical application.

In summary, we have demonstrated that the procedure for ESD and its outcomes can be standardized and enhanced using the MASTER robot, and produced comparable results between ESD experts and non-experts. We also demonstrated that MASTER enabled novices to learn and perform simple ESD procedures to acceptable standards after a short training period.
Competing interests: Philip WaiYan Chiu received honoraria as a speaker from Olympus Co Ltd and Pentax Co Ltd. SJ Phee is cofounder of EndoMaster Pte Ltd and developer of the MASTER robot. Kiyokazu Nakajima served as scientific advisory board member of EndoMaster Pte Ltd. Khek Yu Ho served as co-founder and board member of EndoMaster Pte Ltd and consultant for Mauna Kea Technology Co Ltd. The remaining authors had no conflict of interest to declare.

\section{Institutions}

' CUHK Jockey Club Minimally Invasive Surgical Skills Center, Department of Surgery, The Chinese University of Hong Kong, Hong Kong SAR, China ${ }^{2}$ School of Mechanical and Aerospace Engineering, Nanyang Technological University, Singapore

${ }^{3}$ Solent Centre for Digestive Diseases, Queen Alexandra Hospital, Portsmouth, UK

${ }^{4}$ Department of Gastroenterology and Hepatology, The Jikei University School of Medicine, Tokyo, Japan

${ }^{5}$ Department of Surgery, Osaka University Graduate School of Medicine, Osaka, Japan

${ }^{6}$ Department of Medicine, Yong Loo Lin School of Medicine, National University of Singapore, Singapore

Division of Gastroenterology and Hepatology, University Medical Cluster, National University Health System, Singapore, Singapore

\section{References}

1 WHO. Cancer Incidence and Mortality Worldwide. International Agency for Research on Cancer (IARC). GLOBOCAN 2008

2 Chiu PW. Novel endoscopic therapeutics for early gastric cancer. Clin Gastroenterol Hepatol 2014; 12: 120-125

3 Watanabe K, Ogata S, Kawazoe S et al. Clinical outcomes of EMR for gastric tumors: historical pilot evaluation between endoscopic submucosal dissection and conventional mucosal resection. Gastrointest Endosc 2006; 63: 776- 782

4 Oka S, Tanaka S, Kaneko I et al. Advantage of endoscopic submucosal dissection compared with EMR for early gastric cancer. Gastrointest Endosc 2006; 64: 877-883

5 Choi IJ, Kim CG, Chang HJ et al. The learning curve for EMR with circumferential mucosal incision in treating intramucosal gastric neoplasm. Gastrointest Endosc 2005; 62: 860 - 865

6 Teoh AY, Chiu PW, Wong SK et al. Difficulties and outcomes in starting endoscopic submucosal dissection. Surg Endosc 2010; 24: 1049-1054

7 Ho KY, Phee SJ, Shabbir A et al. Endoscopic submucosal dissection of gastric lesions by using a Master and Slave Transluminal Endoscopic Robot (MASTER). Gastrointest Endosc 2010; 72: 593-599

8 Phee SJ, Reddy N, Chiu PW et al. Robot-assisted endoscopic submucosal dissection is effective in treating patients with early-stage gastric neoplasia. Clin Gastroenterol Hepatol 2012; 10: 1117-1121

9 Sung JJ, $\mathrm{Ng} E K$, Lin JT et al. Digestive cancer management in Asia: position statements: A report on GI Oncology Summit in 2011. J Gastroenterol Hepatol 2012; 27: 1417-1422

10 Everett SM, Axon AT. Early gastric cancer in Europe. Gut 1997; 41: 142 150

11 Gotoda T, Yanagisawa A, Sasako $M$ et al. Incidence of lymph node metastasis from early gastric cancer: estimation with a large number of cases at two large centers. Gastric Cancer 2000; 3: 219-225

12 Probst A, Golger D, Arnholdt $H$ et al. Endoscopic submucosal dissection of early cancers, flat adenomas and submucosal tumors in the gastrointestinal tract. Clin Gastroenterol Hepatol 2009; 7: 149-155

13 Porpiglia F, Morra I, Lucci Chiarissi M et al. Randomised controlled trial comparing laparoscopic and robot-assisted radical prostatectomy. Eur Urol 2013; 63: 606-614

14 Wang Z, Phee SJ, Lomanto $D$ et al. Endoscopic submucosal dissection of gastric lesions by using a master and slave transluminal endoscopic robot: an animal survival study. Endoscopy 2012; 44: 690-694 